(C) 2016 IEEE. Personal use of this material is permitted. Permission from IEEE must be obtained for all other uses, in any current or future media, including reprinting/republishing this material for advertising or promotional purposes, creating new collective works, for resale or redistribution to servers or lists, or reuse of any copyrighted component of this work in other works 


\section{Deployment of Churn Prediction Model in Financial Services Industry}

\author{
Charles Chu \\ Marketing, CFS, Sydney, Australia \\ Charles.Chu@cba.com.au \\ James Brownlow \\ Marketing, CFS, Sydney, Australia \\ James.Brownlow@cba.com.au
}

\author{
Guandong $\mathrm{Xu}^{*}$ \\ Advanced Analytics Institute, UTS, Australia \\ Guandong.Xu@uts.edu.au \\ $\mathrm{Bin} \mathrm{Fu}$ \\ Advanced Analytics Institute, UTS, Australia \\ Bin.Fu@student.uts.edu.au
}

\begin{abstract}
Nowadays, data analytics techniques are playing an increasingly crucial role in financial services due to the huge benefits they bring. To ensure a successful implementation of an analytics project, various factors and procedures need to be considered besides technical issues. This paper introduces some practical lessons from our deployment of a data analytics project in a leading wealth management company in Australia. Specifically, the process of building a customer churn prediction model is described. Besides common steps of data analysis, how to deal with other practical issues like data privacy and change management that are encountered by many financial companies are also introduced.
\end{abstract}

Keywords-customer churn, wealth management, analytics

\section{INTRODUCTION}

In Australia, wealth management businesses act as service providers for individuals and companies to invest in various types of funds, including individual Superannuation \& Pension funds. Currently, the market is highly competitive since customers can transfer from one company to another freely. Therefore, effective customer churn prediction and retention strategies are crucial to maintain high business growth and performance in such a volatile and competitive market. To predict the possible churn customers, various data analytics techniques can be applied to build the predictive models [1,2]. Although these model could generate good prediction, there are still other challenges in practice. For example, business people might also expect to identify customer segments and attributes that leads to a churn, and understand the effectiveness of marketing treatmentsas an added layer of sensitivity and complexity.

In this paper, we focus on presenting our approach to deploy complex modelling into a real business environment. It covers key milestones experienced in the attrition model design and change management, and the cultural shift in leads generation and retention strategy treatment for various channels of customers in a real industry study.

\section{RELATED WORK}

There has been increasing applications of predictive analytics in different industries $[3,4]$. Churn prediction is generally treated as a binary classification problem. Various techniques have been applied such as sampling, model selection and boosting approaches [5, 6, 7]. However, no papers were identified that discuss the milestones involved when introducing change management to a financial industry.

\section{MODEL DEPLOYMENT}

This section introduces major concerns of practical model deployment, and the approaches we adopt to deal with accordingly.

\section{A. Model Building}

Our implementation of building models consists of several major phases, including: data preparation, feature engineering, model evaluation and selection etc. To capture as much useful information as possible, we create a comprehensive set of features by exploiting heterogeneous data sources, like user demographic, transaction, fund performance, adviser and dealer profiles, etc.

We firstly built 4 models include Logistic regression, Naive Bayesian, Support Vector Machine and Random Forests based on finding the balance between prediction accuracy and the ease for business to act on the insights. It is absolutely imperative for the business to be able to read and understand what makes a customer at risk of churn. It is with this knowledge the business is able to segment customers into different types of channels and marketing interventions. With this in mind, whilst Logistic regression generates a very insightful output for business to understand, the sacrifice to prediction accuracy made us shy away from selecting this model.

We have built aforementioned 4 models for superannuation and pension products separately. Figure 1 and 2 give the performance comparison. Random forest was selected as the best model given its performance and the ability to output attribute importance for the business to produce relevantly tailored communication themes and approaches.

\section{B. Risk Measurements}

Data sensitivity is the most important measure before deployment. This includes masking customer information to lose 
original meaning while keeping analytics value and removal of attributes that can lead to leakage of confidential customer information. In that regard, it is imperative to consult Legal and Risk departments to ensure accurate data classification and handling processes are in place to ensure the risk of unwanted privacy and brand issues is not inflated. Other risk measures include ongoing flight risk, key person risk, data storage risk and data access risk.

\section{Financial intermediaries}

Demonstrating how the analytics output benefits financial advisers is the most important task. Using the output from the predictive models, we were able to create a customized attribute chart that introduces portfolio insights and visually captures top attributes that drive attrition. This is business intelligence that has never been available to financial advisers.

Customers were segmented based their demographics and attrition modelling output. Approvals were given to test and learn the effectiveness of retention treatments on each of these segmented deciles. Customers with high attrition risk will receive a retention message that considers their demographics and past account behaviors; customers with lower attrition score will, instead of receiving a retention campaign, receive upsell/cross-sell marketing material. The aim is to evaluate the real business application and benefit of the model output by comparing test and learn outcome against control groups.

\section{Measure of success}

Both internal stakeholders and Financial Intermediaries are extremely cautious when it comes to the quality of campaigns to the end customers. Due to the fast paced nature of the financial market, additional out of time monitoring took place to provide additional confidence in the model. A forecast of potential business profit was also produced to spark additional commitment. Based on current portfolio sizes, revenue figures were graphed based on projected campaign success.

Further, it is important to identify and connect high importance model attributes back into key business strategies/ objectives. It immediately generates the desire to dedicate resources into the retention initiative.

\section{RESULTS}

Leadership approval and funding was secured off the back of our approach to deploying machine learning outputs. Using appropriate analytics transformations, we were able to successfully turn complex data and modelling output into potential business strategies. One example of real time model result is shown in Figure 3. Here, all the super accounts are ranked in terms of their churn porobability predicted by model, and divided into 10 deciles. It is clear that more real churn customers are captured in top deciles, meaning our models perform well.

\section{CONCLUSION}

This paper introduces the process of deploying a data analysis project in a financial services company, and some issues required to be addressed in practice. While the leadership team always understood the importance of incorporating predictive analytics, it was not clear to them how it could be done. Often though, even though a machine learning model can generate predictive outputs, without careful change management or an appropriate plan to leveraging this intelligence and taking action on the predictions, the consequences can potentially become even more costly to the business than if they had no predictions.

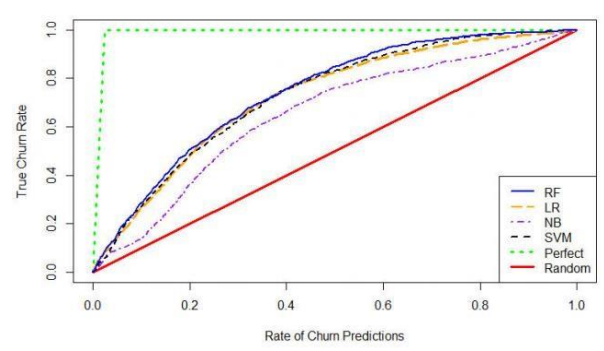

Figure 1 - Prediction performance of super model

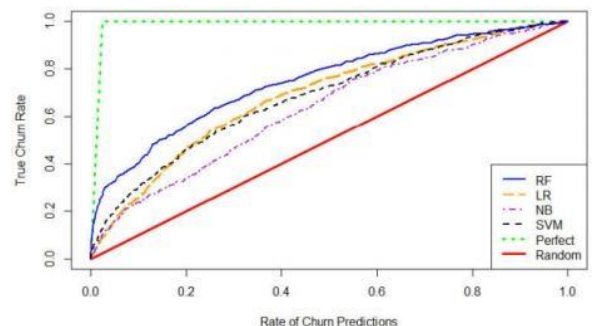

Figure 2 - Prediction performance of pension model

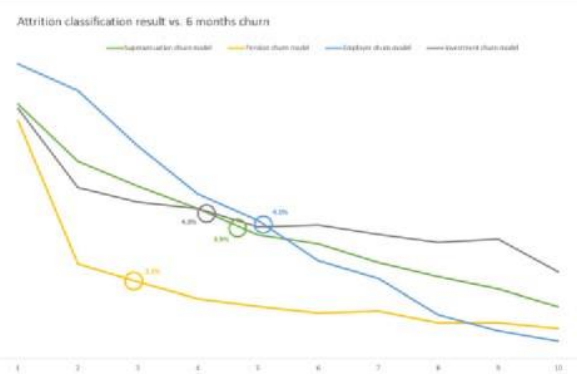

Figure 3 - Live performance of various models

\section{REFERENCES}

[1] L. Breiman, Random forests Machine learning, 45(1):5-32, 2001

[2] K. Coussement and K. W. De Bock, Customer churn prediction in the online gambling industry: The beneficial effect of ensemble learning. Journal of Business Research, 66(9):1629-1636, 2013.

[3] G. Dror, D. Pelleg, O. Rokhlenko, and I. Szpektor, Churn prediction in new users of yahoo! answers. In Proceedings of the 21st International Conference Companion on World Wide Web, pages 829-834, 2012

[4] Y. Huang, F. Zhu, M. and Yuan, et al. Telco churn prediction with big data. In Proceedings of the 2015 ACM International Conference on Management of Data, pages 607-618, 2015.

[5] N. Lu, H. Lin, J. Lu, and G. Zhang, A customer churn prediction model in telecom industry using boosting. IEEE Transactions on Industrial Informatics, 10(2):1659-1665, 2014.

[6] ] V. Mahajan, R. Misra, and R. Mahajan, Review of data mining techniques for churn prediction in telecom. Journal of Information and Organizational Sciences, 39(2):183-197, 2015.

[7] P. Rothenbuehler, J. Runge, F. Garcin, and B. Faltings, Hidden markov models for churn prediction. In Proceedings of the SAI Intelligent Systems Conference, pages 723-730, 2015. 